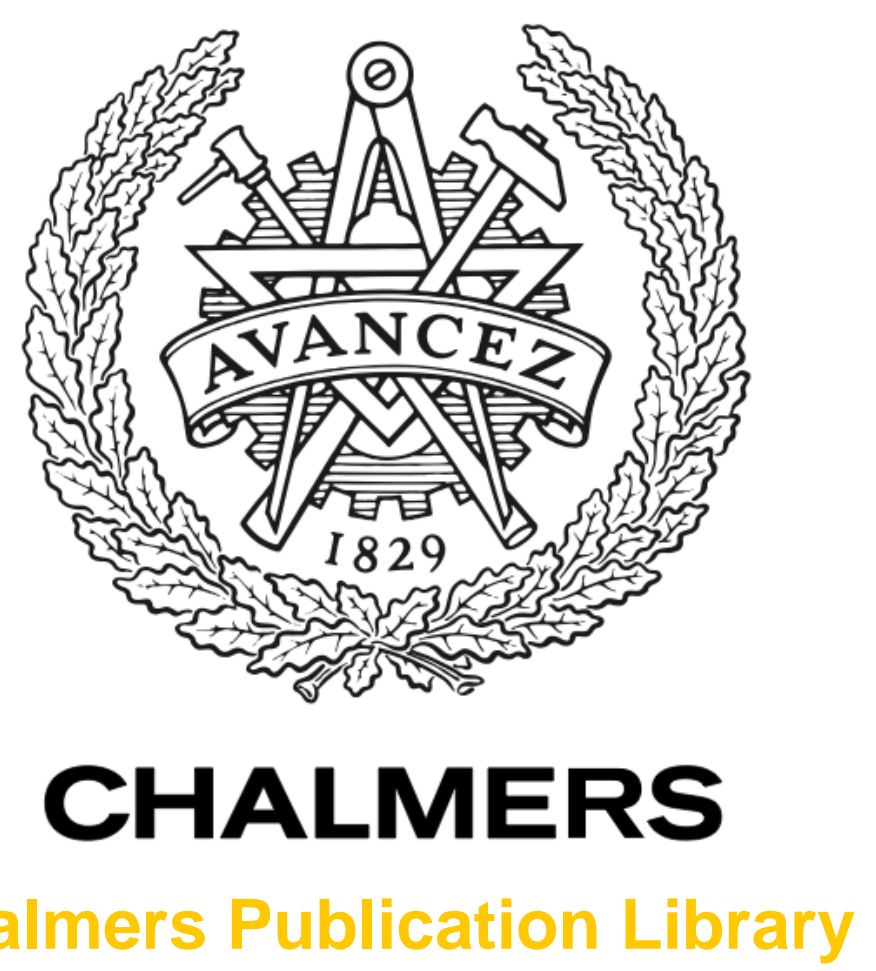

Chalmers Publication Library

Source Modeling using Phaseless Low-Frequency Near-Field Measurements

This document has been downloaded from Chalmers Publication Library (CPL). It is the author's version of a work that was accepted for publication in:

IEEE Transactions on Electromagnetic Compatibility (ISSN: 0018-9375)

Citation for the published paper:

Johansson, M. ; Lui, H. ; Bolomey, J. (2012) "Source Modeling using Phaseless Low-

Frequency Near-Field Measurements". IEEE Transactions on Electromagnetic Compatibility, vol. 54(3), pp. 613-624.

http://dx.doi.org/10.1109/TEMC.2011.2171056

Downloaded from: http://publications.lib.chalmers.se/publication/149987

Notice: Changes introduced as a result of publishing processes such as copy-editing and formatting may not be reflected in this document. For a definitive version of this work, please refer to the published source. Please note that access to the published version might require a subscription.

Chalmers Publication Library (CPL) offers the possibility of retrieving research publications produced at Chalmers University of Technology. It covers all types of publications: articles, dissertations, licentiate theses, masters theses, conference papers, reports etc. Since 2006 it is the official tool for Chalmers official publication statistics. To ensure that Chalmers research results are disseminated as widely as possible, an Open Access Policy has been adopted.

The CPL service is administrated and maintained by Chalmers Library. 


\title{
Source Modeling Using Phaseless Low-Frequency Near-Field Measurements
}

\author{
Markus Johansson, Hoi-Shun Lui, Member, IEEE, Jean-Charles Bolomey, Fellow, IEEE, \\ and Mikael Persson, Member, IEEE
}

\begin{abstract}
Field measurements of both amplitude and phase generally are more complicated and require more expensive equipment than amplitude-only measurements. Phase retrieval from measured phaseless field data is, therefore, of interest for source modeling in dosimetry applications, electromagnetic compatibility investigations, near-field to far-field transformations and antenna diagnostics. We here present a phase-retrieval method that uses an optimization algorithm based on the phase angle gradients of a functional. Numerical test cases have shown that the method is working for different initial phase distributions as well as different placements of the source. The method also works well for a test case with measured $50 \mathrm{~Hz}$ magnetic flux density from a transformer. The obtained phase angles on a measurement plane in front of the transformer gave calculated field amplitudes on other measurement planes that agree well with measured field. The ratios between the largest amplitude difference and the largest measured amplitude for the three Cartesian magnetic flux density components, for one of the planes, are for example $6.62 \%, 9.51 \%$ and $6.40 \%$.
\end{abstract}

Index Terms-Complex source modeling, electromagnetic dosimetry, electromagnetic measurements, inverse problem, optimization methods.

\section{INTRODUCTION}

$\mathbf{P}$ HASE retrieval from measured phaseless field data is of interest for near-field to far-field transformations [1], antenna diagnostics [2], as well as for dosimetry applications [14]. Phase-retrieval methods are interesting, since field measurements of both amplitude and phase generally require more efforts and are more expensive than amplitude-only measurements. Furthermore, they are particularly important for electromagnetic compatibility (EMC) applications involving incoherent emissions from nonintentional parasitic sources.

In dosimetry studies, it is important to be able to model field distributions from electromagnetic sources, to make it possible to predict whether exposure safety guidelines are complied with. If the total complex field, both amplitude and phase, is known on

M. Johansson, H.-S. Lui, and M. Persson are with the Chalmers University of Technology, Göteborg SE-412 96, Sweden (e-mail: markusjo@chalmers.se; lui@ieee.org; mikael.persson@chalmers.se).

J.-C. Bolomey is with Laboratoire des Signaux et Systèmes, Département de Recherche en Electromagnétisme, Supelec, Gif-sur-Yvette, 91192, France (e-mail: jean-charles.bolomey@ supelec.fr).

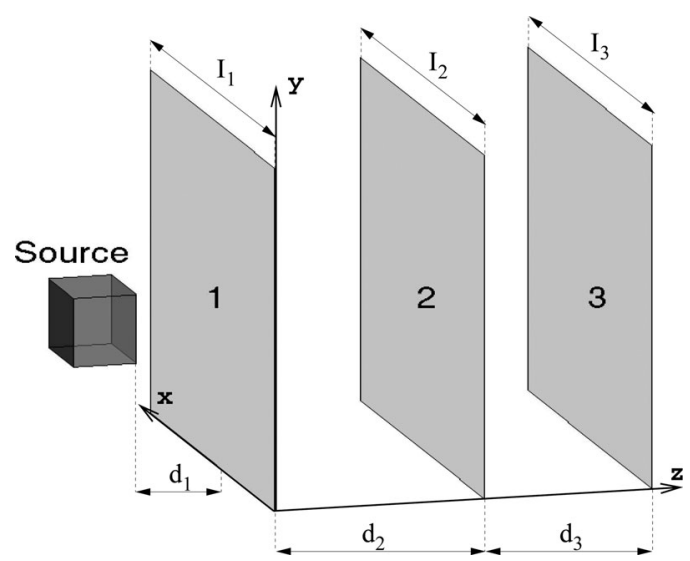

Fig. 1. Source in front of planes where field amplitudes can be measured.

a surface that encloses an electromagnetic source in free space, the field outside the surface can be calculated according to the field equivalence principle [15]. The field in an area in front of the source can be calculated with a good accuracy provided the total field is known on a large enough planar surface between the source and the area of interest. However, usually only the amplitude values of the field are measured by dosimetric probes. Therefore, a method that can retrieve the phase from measured field amplitudes on a set of parallel planes close to the source is needed (see Fig. 1).

Different phase-retrieval methods have been presented in the scientific literature [1]-[13]. Some of them [1]-[5] are gradientbased and try to minimize a function, whereas other methods [6]-[9] aim to recreate the phase angles in an iterative fashion, by propagating field estimates back and forth between different surfaces. Hybrid algorithms, which combine more than one method, have also been presented [10], [11].

A formulation of the problem of determining radiation pattern, from phaseless square amplitude measurements over two planes, as a quadratic inverse problem, is described by Isernia et al. [1]. The radiated field in the near zone is searched for using a gradient based functional minimization. The method in [1] was experimentally tested with near-zone data from a shaped reflector at $9 \mathrm{GHz}$ and the obtained radiation pattern was similar to the calculated radiation pattern using a standard near-field technique. Isernia et al. [3] have also discussed a method that determines radiation pattern from phaseless nearfield data collected over limited domains on planar surfaces by minimization of a functional. Strategies to avoid getting trapped in local minima is discussed both in [1] and by Soldovieri et al. in [5]. In a method presented by Sarkar et al. [4] and Las-Heras 
et al. [2], an expansion of an equivalent magnetic current distribution on a plane in front of the source is done. To find the correct equivalent current, expansion coefficients that minimize a cost function are searched for with a gradient-based algorithm. The measurement points can be on planes, but can also be on any other arbitrary surface. Roczniak et al. [13] have presented a procedure that uses measured amplitude distributions on spherical surfaces and searches for coefficients for a spherical wave expansion, that minimize a cost function.

In optics, phase-retrieval by propagating field estimates back and forth between different planes, using fast Fourier transform (FFT), was put forward by Gerchberg and Saxton [6]. Between consecutive propagations in the algorithm, the field amplitudes are reset to the measured ones. In [12], the Gerchberg-Saxton algorithm and related algorithms for phase-retrieval and also gradient search methods are discussed by Fienup. After these early works in optics, other researchers have used similar methods for phase-retrieval in the antenna community [7]-[9].

An iterative Fourier procedure for phase-retrieval in a forward-backward fashion, used for constructing the far-field pattern and performing diagnostic of an antenna under test, has been presented by Yaccarino et al. [8]. In a slightly modified version, Farhad Razavi et al. [10] use the differential evolutionary algorithm to search a suitable initial guess for improving the convergence of the iterative reconstruction. A cylindrical phaseretrieval algorithm, which propagates field estimates back and forth and uses field intensity measurements made on two cylindrical surfaces instead of planes, has been described by Tennant et al. [7].

Phase-retrieval methods are useful for numerical dosimetry at both high and low frequencies. For instance, a method similar to the iterative procedure in [8], has been used by Fridén et al. [9] to retrieve the electric field distribution and determine the specific absorption rate (SAR) in a phantom with lossy liquid. The method suffers from a stagnation problem. To improve the convergence, a three-step algorithm that also utilizes a gradient search method, has been proposed by Fridén et al. [11]. Phase-retrieval for low-frequency electromagnetic field is also of interest for electromagnetic compatibility (EMC) investigations. Source reconstruction for electromagnetic interference (EMI) studies, using both field amplitude and phase, is described in [16]-[18]. It is reported by Rodríguez et al. [16] that phase retrieval was first attempted in their proposed system, but the results were not accurate. The authors concluded that this was due to the long wavelengths of the radiated field. Baudry et al. [19] discussed near-field techniques in EMC investigations and presented results for a method in which equivalent electric dipoles are used for source modeling. It may be interesting to mention that similar low-frequency inverse source problems are relevant for other fields of applications such as medical imaging by magnetoencephalography (MEG) [20] or magnetization identification [21].

As described above, results from earlier attempts to do phaseretrieval for low frequencies were not encouraging. From the above discussion, it is clear that if phase-retrieval can be performed even for situations with distances between the measurement planes that are small in terms of wavelengths, it is interesting not only for numerical dosimetry, but also for EMC applications.

It is reported in [1] that the distance between the scanning surfaces plays an important role in determining the lack or occurrence of local minima. Both theoretical investigations and experimental results are presented. A practical procedure for antenna testing is also described in which the distance between the scanning surfaces is chosen to be of the order of a few wavelengths. As compared to [1], the method we have employed, which is further described in next section, is also gradient based and a functional is minimized using the steepest descent method.

However, an important difference between the two methods is the choice of unknowns. In [1] the complex field is searched for and the functional is formulated so that the inverse problem becomes quadratic. In the method we use, namely the phase angle gradient method (PAGM), we instead search for the correct phase angles that together with measured field amplitudes on one measurement plane, give correct calculated field amplitudes on two other planes. In other words, the unknowns that we are searching for in the PAGM are the phase angles, but not the complex fields. The tangential component of the electric field on the measurement plane closest to the source $\bar{E}_{t a n}$ can be written as an expression containing the measured amplitudes and sinusoidal functions of the unknown phase. The field on the other planes can be written in terms of $\bar{E}_{t a n}$, which is discussed further in next section. This leads to an inverse problem with a nonlinearity that is not quadratic. So although the basic goal for the PAGM and the method in [1] are to find the correct field from amplitude measurements, they are quite different mathematically. Thus the results in [1] do not provide information about whether the PAGM works for cases with plane distances much smaller than the wavelength or not. The PAGM has previously been presented and tested for numerical high frequency cases by Johansson et al. [14]. Later the PAGM was extensively tested with various numerical test cases in [22], [23]. It was found that the method performed well for test cases with separations between the measurement planes ranging from $0.4 \lambda$ down to separations in the scale of $10^{-9} \lambda$.

This paper is more particularly focused on low frequency applications for which no simple guidelines are available to dimension the experimental setup. While such guidelines exist for microwave antenna testing in terms of sampling rate, truncation errors, distance of measurement, etc. they are intimately related to the wavelength, and, hence, of no practical use at low frequencies. To demonstrate its effectiveness at low frequencies and to obtain some guidelines to define the experimental setup, the PAGM is here tested for determining the $50-\mathrm{Hz}$ field distribution around a transformer. To show that we can do phase retrieval for cases with relevant distances (i.e., sampling space and plane-to-plane separation distance) much smaller than the wavelength, we have also considered different numerical test cases.

An important advantage with the PAGM is that it can be used for many different electromagnetic sources, without the need for modeling of all the details of the sources. In the source reconstruction studies described in [16]-[18], both field amplitude and phase were used. For the PAGM, on the other hand, it is 
enough to measure the field amplitudes on a set of planes in front of each source. The PAGM is versatile, since it can be used for both high and low frequencies. In particular it works well even for cases with plane sizes and separations between the planes that are extremely small in terms of wavelengths. As compared to the source modeling method in [19], where the equivalent sources are found through successive comparison between the model predictions and the measurements, the PAGM is more automated and the equivalent sources do not need to be searched manually.

The rest of the paper is organized as follows. Section II describes the phase-retrieval method we have used in more detail. The Sections III and IV contain results for test cases with numerical and measured field values respectively. In Section V conclusions are presented.

\section{The Phase Angle Gradient Method}

According to the field equivalence principle, the actual source in Fig. 1 can be replaced by an equivalent magnetic surface current density on plane 1

$$
\bar{M}_{s}=-2 \hat{n} \times \bar{E}_{p 1}
$$

where $\hat{n}$ is a unit vector perpendicular to plane 1 pointing toward the other planes and $\bar{E}_{p 1}$ is the total electric field on plane 1 .

One can divide plane 1 into a square grid with the grid cell area $\Delta S=\Delta x \cdot \Delta y$, where $\Delta x$ and $\Delta y$ are the sampling spacing in $x$ - and $y$-directions, respectively. If the point in the middle of the square number $p$ is represented by $\bar{r}_{p}^{\prime}$, the Cartesian components of the electric field $\bar{E}$ in point $\bar{r}$ can be calculated [14] with the expressions

$$
\begin{aligned}
E_{x}(\bar{r})= & -\frac{\Delta S}{2 \pi} \sum_{p} E_{x}\left(\bar{r}_{p}^{\prime}\right) \frac{\partial G\left(\bar{r}, \bar{r}_{p}^{\prime}\right)}{\partial z} \\
E_{y}(\bar{r})= & -\frac{\Delta S}{2 \pi} \sum_{p} E_{y}\left(\bar{r}_{p}^{\prime}\right) \frac{\partial G\left(\bar{r}, \bar{r}_{p}^{\prime}\right)}{\partial z} \\
E_{z}(\bar{r})= & \frac{\Delta S}{2 \pi} \sum_{p}\left(E_{x}\left(\bar{r}_{p}^{\prime}\right) \frac{\partial G\left(\bar{r}, \bar{r}_{p}^{\prime}\right)}{\partial x}\right. \\
& \left.+E_{y}\left(\bar{r}_{p}^{\prime}\right) \frac{\partial G\left(\bar{r}, \bar{r}_{p}^{\prime}\right)}{\partial y}\right) .
\end{aligned}
$$

Here, the system of coordinates is chosen such that plane 1 is part of the $z=0$-plane, as illustrated in Fig. 1 , and $G\left(\bar{r}, \bar{r}^{\prime}\right)$ is the Green's function

$$
G\left(\bar{r}, \bar{r}^{\prime}\right)=\frac{e^{-j k\left|\bar{r}-\bar{r}^{\prime}\right|}}{\left|\bar{r}-\bar{r}^{\prime}\right|}
$$

where $k$ is the wavenumber. Note that for the special case when the wavelength is much larger than the relevant distances, the exponential function in (5) can be approximated with 1. If plane 1 is chosen large enough and $\Delta S$ small enough, the (2)-(4) give a good approximation for the electric field on the planes 2 and 3. The required size of plane 1 depends on several factors. The plane should be large enough so that the contributions to the calculated field from the field outside the edges can be neglected, but how large that is depends on where the area of interest for the field calculation is. A larger plane may for example be needed, if the field further away from the plane is wanted. The requirement on the plane can also vary, if the area of interest is in the center in front of the plane or more towards the edges. Moreover, the resolution need to be high enough to resolve the spatial variation of the field. Thus, it is hard to formulate a simple general condition for required plane size and resolution. To give an insight in what a suitable plane size can be, it is investigated in Section III-D with numerical calculations in how large volume the field can be obtained with good accuracy for a given plane size. The requirement for the resolution of the measured field is explored in IV-A. Since the field amplitudes are known on plane 1 , the field on the other planes can be regarded as a function of the unknown phase angles of the tangential components of $\bar{E}$ on plane 1 .

After the phase angles on plane 1 have been initiated, the resulting field estimates on the planes 2 and 3 can be calculated. To find the correct phase, the initial angles are altered in small steps, so that the field amplitudes $\left|E_{i}\right|_{n}$, where $\mathrm{n}$ is a computational grid point on plane 2 or 3 , converge to the measured values $\left|E_{i}^{m}\right|_{n}$. A functional $J$ of the phase can be defined as

$$
\begin{aligned}
J \equiv & \frac{1}{2} \sum_{n}\left(\left(\left|E_{x}\right|_{n}-\left|E_{x}^{m}\right|_{n}\right)^{2}+\left(\left|E_{y}\right|_{n}-\left|E_{y}^{m}\right|_{n}\right)^{2}\right. \\
& \left.+\left(\left|E_{z}\right|_{n}-\left|E_{z}^{m}\right|_{n}\right)^{2}\right) .
\end{aligned}
$$

The phase angles are changed in the opposite direction of the phase angle gradients of $J$, so that $J$ is minimized. That is, in each iteration the phase angles for the $x$ - and $y$-components of the field in each grid cell on plane 1 are updated according to the equation

$$
\phi^{\text {new }}=\phi^{\text {old }}-G_{\phi}^{\text {old }} \cdot \alpha
$$

where $\phi^{\text {old }}$ and $\phi^{\text {new }}$ are the values of a phase angle $\phi$ before and after the update. $G_{\phi}^{\text {old }}$ is

$$
G_{\phi}=\frac{\partial J}{\partial \phi}
$$

calculated for the values of the phase angles on plane 1 before the update and $\alpha$ is a constant that is chosen with a line-search method. The iterative procedure is finished after an adjustable number of iterations. To verify that a sufficient number of iterations have been performed, the convergence of the procedure should be checked by plotting $J$ as function of iteration number and it should also be made sure that amplitudes calculated with the retrieved phase agree well with the measured amplitudes.

The measurement points could be placed in many different ways. For simplicity, the measurement points have been located on a set of parallel planes, with the same distance between the measurement points on all the planes. Different numbers of measurement planes can be used in our implementation of the PAGM, but experience has shown that it is suitable to use three measurement planes to get a reliable and accurate result. The PAGM has performed well with three measurement planes for many different test cases, with plane sizes and separations 


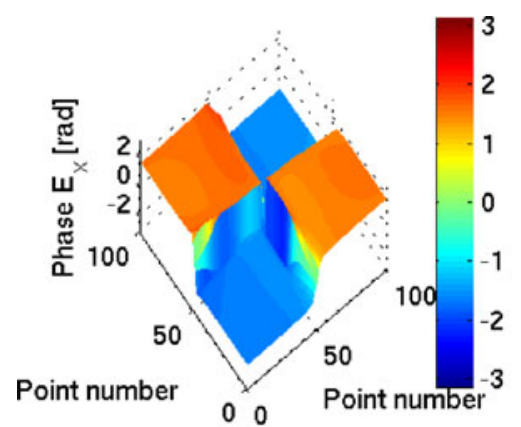

(a)

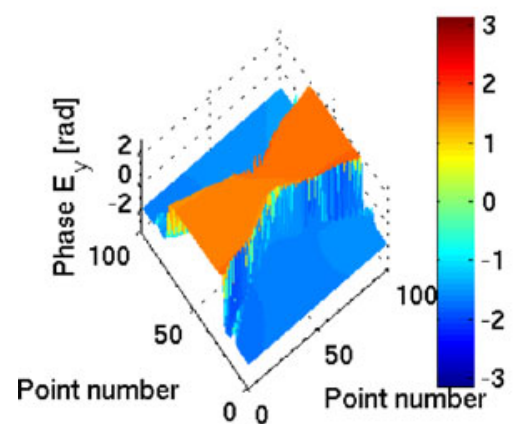

(d)

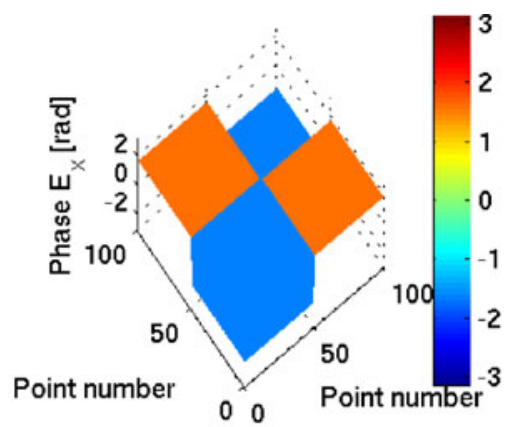

(b)

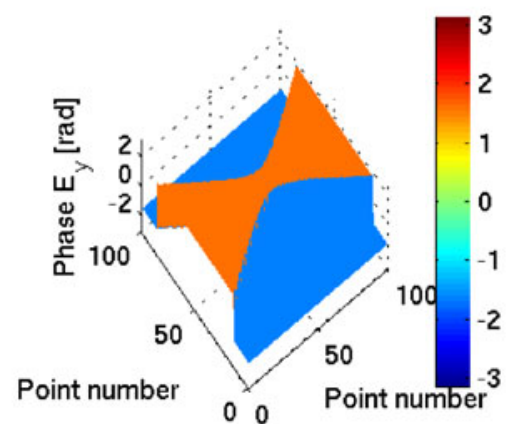

(e)

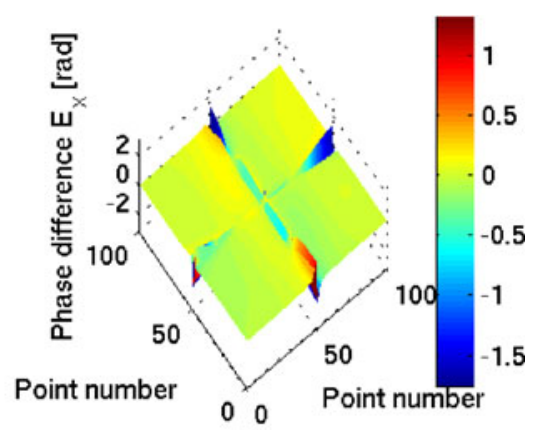

(c)

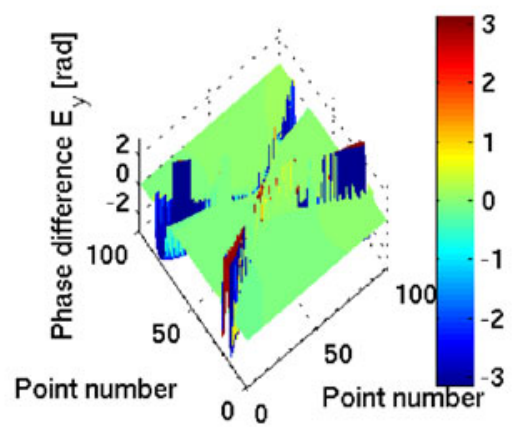

(f)

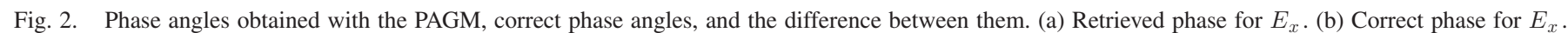

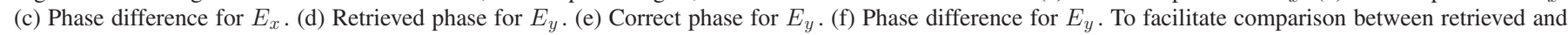

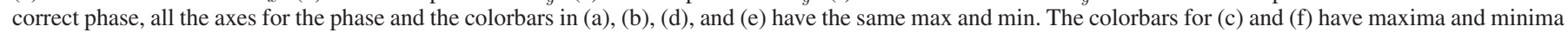
that are chosen to make it possible to see how the differences vary.

between the planes extremely small in terms of wavelengths as well as comparable to the wavelength [22], [23].

Although the PAGM originally was developed for calculation of the phase angles of the electric field from measured electric field amplitudes, it can, because of the symmetry of Maxwell's equations, also be used to calculate the phase angles of the magnetic flux density $\bar{B}$ from measured amplitudes of $\bar{B}$.

\section{Results From Synthetic ANALYTICAl DATA}

\section{A. Analytical Test Case With Infinitesimal Dipole}

In the first example, field values calculated with an analytical formula for a 50-Hz $y$-directed infinitesimal dipole were utilized to test the PAGM. Calculated field amplitudes for three vertical parallel planes in front of the source (see Fig. 1), were used to calculate the phase angles. The infinitesimal dipole source was centered and the right-angled distance between the centre of the plane closest to the source, plane 1, and the infinitesimal dipole was $d_{1}=5 \mathrm{~cm}$. On plane 1 , field values from $100 \times$ 100 measurement points were used and on the other planes $60 \times 60$ measurement points were used. Plane 1 was chosen larger than the other planes, in order to increase the accuracy in the calculations of the field on the other planes. The distance between the points on each plane in $x$ - and in $y$-directions was $\Delta x=\Delta y=0.5 \mathrm{~cm}$. Thus, the widths of the planes 1,2 , and 3 became $I_{1}=50 \mathrm{~cm}, I_{2}=30 \mathrm{~cm}$ and $I_{3}=30 \mathrm{~cm}$. From plane 1 to plane 2 and from plane 2 to plane 3 the distances were $d_{2}=$ $5 \mathrm{~cm}$ and $d_{3}=2.5 \mathrm{~cm}$, respectively.
Fig. 2 shows the phase angles obtained using the PAGM, with the initial value zero for all the phase angles, and the correct phase angles from the analytical formula, for the field components tangential to the planes, $E_{x}$ and $E_{y}$, on plane 1 . The difference between retrieved and correct phase is also shown in the figure. It can be seen in Fig. 2(a), (b), (d), and (e) that the PAGM gives phase angles that are similar to the correct ones for both the tangential field components. Fig. 2(c) and (f) illustrates that for both the field components the differences between calculated and correct phase angles are small, with the exception of some unimportant larger errors in areas near rapid changes in the phase.

For here considered test case, the relevant distances are so small compared to the wavelength that we do not expect any observable phase shift due to propagation. There are, however, $\pi$ rad jumps in the phase, where the field components change sign and take opposite directions. The large phase errors that can be observed in Fig. 2 near those phase jumps are not important, since the amplitudes of the field components near the places for the sign changes are small. It is also reasonable that the phase angle errors in such places are larger, since the errors in points where the amplitudes are small do not change the minimized functional much.

\section{B. Different Initial Phase Distributions}

To investigate whether the PAGM works well for different initial values for the phase angles, the test case was run for some different initial values. Fig. 3 shows the functional $J$ as function 


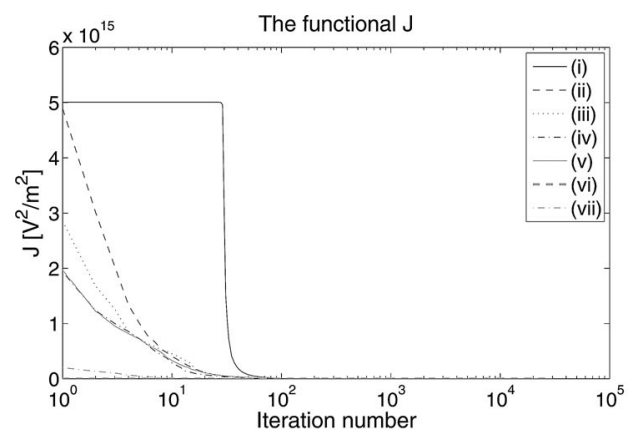

(a)

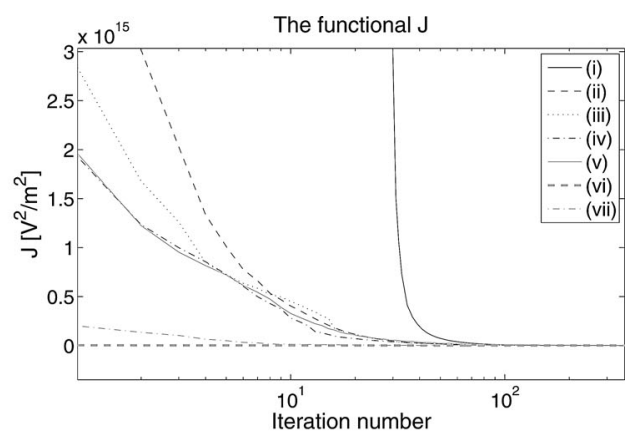

(b)

Fig. 3. Functional $J$ as function of iteration number for seven different initial phase distributions. (a) The whole plot. (b) Zoomed in plot. (i) initial phase zeros, (ii) random interval $-0.04 \pi$ rad to $0.04 \pi$ rad around zero phase, (iii) random interval $-0.2 \pi$ rad to $0.2 \pi$ rad around zero phase, (iv) initial phase random, (v) initial phase random, (vi) random interval $-0.1 \pi \mathrm{rad}$ to $0.1 \pi$ rad around calculated phase, and (vii) random interval $-0.5 \pi$ rad to $0.5 \pi$ rad around calculated phase.

of iteration number for seven different initial phase distributions. One distribution with the initial value zero for all the phase angles (i) was used. Random numbers were used in the other distributions. For two of them, (ii) and (iii), the initial phase in each point was a random number in an interval around zero. The two initial phase distributions, (iv) and (v), had random phase. For the last two phase distributions, (vi) and (vii), the initial phase in each point was a random number in an interval around previously calculated phase, that was obtained with initial phase zeros for all the angles. It can be seen in the figure that the curves for the various initial phase distributions start with different values for $J$, but when the number of iterations becomes large enough the curves reach similar values.

To quantify the results for the test case, the summed and weighted phase angle error

$$
\phi_{\text {error }}=\sqrt{\frac{\sum_{n}\left(\left|E_{x}\right|_{n}^{2}\left(\phi_{x, n}^{\text {diff }}\right)^{2}+\left|E_{y}\right|_{n}^{2}\left(\phi_{y, n}^{\text {diff }}\right)^{2}\right)}{\sum_{n}\left(\left|E_{x}\right|_{n}^{2}+\left|E_{y}\right|_{n}^{2}\right)}}
$$

was calculated. Here, $\phi_{x, n}^{\text {diff }}$ is the error in the calculated phase angle for $E_{x}$ in the measurement point on plane 2 or 3 with number $n$ and $\phi_{y, n}^{\text {diff }}$ is the corresponding error for $E_{y}$. The average is weighted with the amplitudes, as it is more important that errors are small in points where the amplitude is large, than in points with small amplitudes. If an unessential constant phase is added to all the obtained phase angles, the resulting phase is neither better nor worse, but the resulting summed and weighted phase angle error can become different. In order to get a fair measure of how good the resulting phase is, the constant phase that gives the smallest $\phi_{\text {error }}$ if added to the calculated phase, is numerically searched for. The $\phi_{\text {error }}$ that is obtained for the found constant phase, when it is added to the calculated phase, is the value that then is used.

The obtained, summed, and weighted phase angle error for the test case as a function of iteration number is shown in Fig. 4. Here, the configurations for the initial phase distribution that were used to get the results in Fig. 3 are considered. Since the two initial phase distributions with random intervals around previously calculated phase were quite close to the correct phase, the weighted phase angle errors for those distributions were rel-

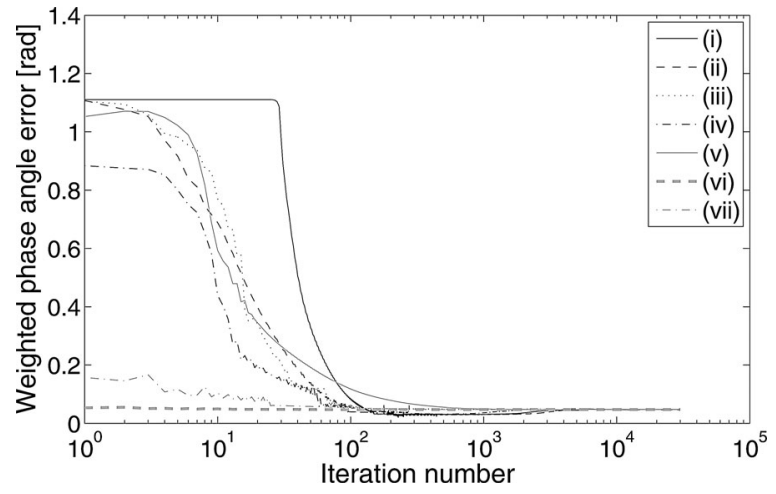

Fig. 4. Obtained, summed, and weighted phase angle error as function of iteration number, for different initial phase distributions: (i) Initial phase zeros, (ii) random interval $-0.04 \pi$ rad to $0.04 \pi \mathrm{rad}$ around zero phase, (iii) random interval $-0.2 \pi$ rad to $0.2 \pi$ rad around zero phase, (iv) initial phase random, (v) initial phase random, (vi) random interval $-0.1 \pi$ rad to $0.1 \pi$ rad around calculated phase, and (vii) random interval $-0.5 \pi \mathrm{rad}$ to $0.5 \pi \mathrm{rad}$ around calculated phase.

atively small already in the beginning. The errors were initially larger for the initial distributions with random phase, random intervals around zero and zero for all the phase angles. It can, however, be seen in Fig. 4 that all the curves reach approximately the same low, summed, and weighted phase angle error that indicates that the method works well for the different initial phase distributions.

\section{Test Case With Off-Centered Dipole}

Since transformers and other electromagnetic sources can be modeled by a set of infinitesimal dipoles, a test case with a centered infinitesimal dipole is a suitable case to start with. However, in order to test the method further and to have an idea of the possible dimensions of the radiating object under test with the previous arrangement of measurement planes, it is also interesting to investigate what happens if the dipole is offcentered. Fig. 5 shows results for a case with the same settings as the case in Section III-A, except that the source was moved $7 \mathrm{~cm}$ in the negative $y$-direction. It can be seen in Fig. 5(a), (b), (d), and (e) that the PAGM gives phase angles that are similar to the correct ones for both the tangential field components. 


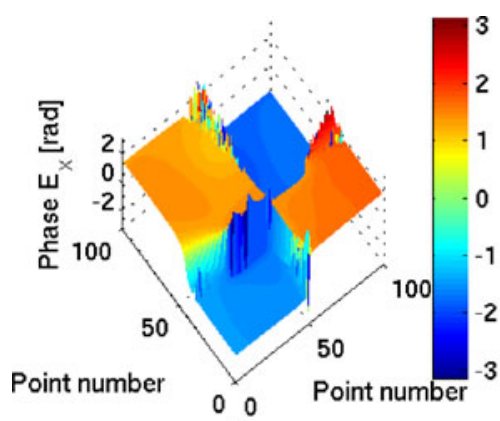

(a)

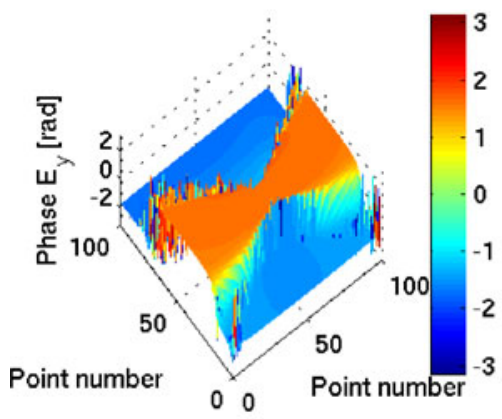

(d)

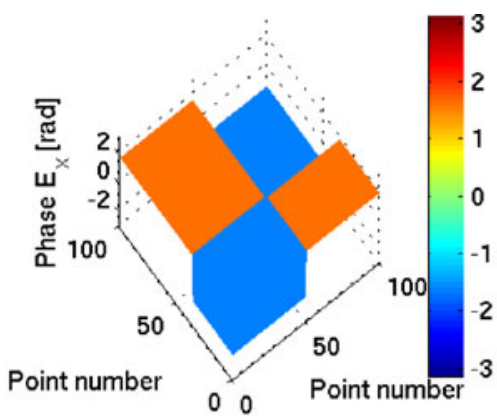

(b)

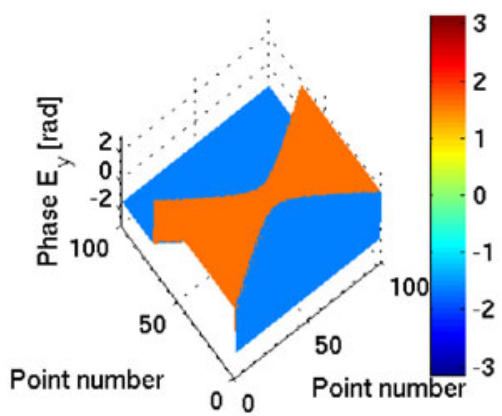

(e)

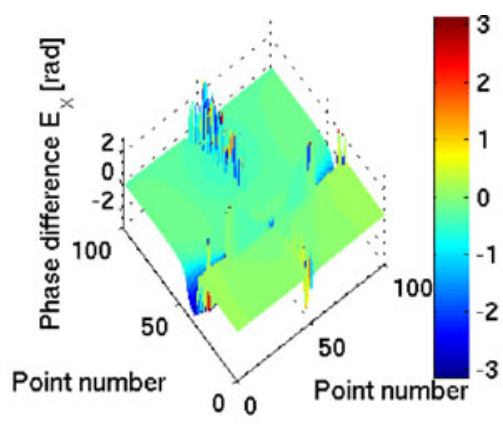

(c)

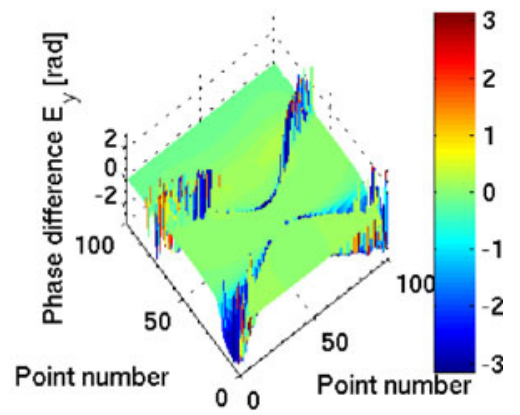

(f)

Fig. 5. Phase angles obtained with the PAGM for a test case with an off-centered infinitesimal dipole, correct phase angles and the difference between them. (a) Retrieved phase for $E_{x}$. (b) Correct phase for $E_{x}$. (c) Phase difference for $E_{x}$. (d) Retrieved phase for $E_{y}$. (e) Correct phase for $E_{y}$. (f) Phase difference for $E_{y}$. To facilitate comparison between retrieved and correct phase, all the axes for the phase and the colorbars in (a), (b), (d), and (e) have the same max and min. The colorbars for (c) and (f) have maxima and minima that are chosen to make it possible to see how the differences vary.

Fig. 5(c) and (f) illustrates that for both the field components the differences between calculated and correct phase angles are small, with the exception of some unimportant larger errors in areas near rapid changes in the phase. The summed and weighted phase angle error was $0.095 \mathrm{rad}$ which is small, but not surprisingly it was larger than the corresponding error for the centered dipole which was 0.048 rad. From these results, it can be anticipated that high accuracy in the phase retrieval can be obtained for centered test objects with dimensions up to at least $15 \mathrm{~cm}$, with the previously defined arrangement of the measurement planes.

\section{Volume Where the Field Can be Obtained}

As the phase has been retrieved on a plane with finite size, it is interesting to consider in how large volume the retrieved phase can give calculated field with good accuracy. Therefore, the field amplitudes on eight planes in front of the source, for the test case in Section III-A, were calculated. The size of each of these planes was $30 \mathrm{~cm} \times 30 \mathrm{~cm}$. The field was calculated using the measured field and the retrieved phase on plane 1 . The separation between the different planes was $5 \mathrm{~cm}$. The average of the absolute value for the amplitude error $A_{\text {average,error }}$ on each of the planes with the size $30 \mathrm{~cm} \times 30 \mathrm{~cm}$ was divided by the largest amplitude on the plane $A_{\max }$. This was done for each Cartesian field component. The resulting error measure can be written

$$
E_{\text {average,error }}=\frac{A_{\text {average,error }}}{A_{\max }} .
$$

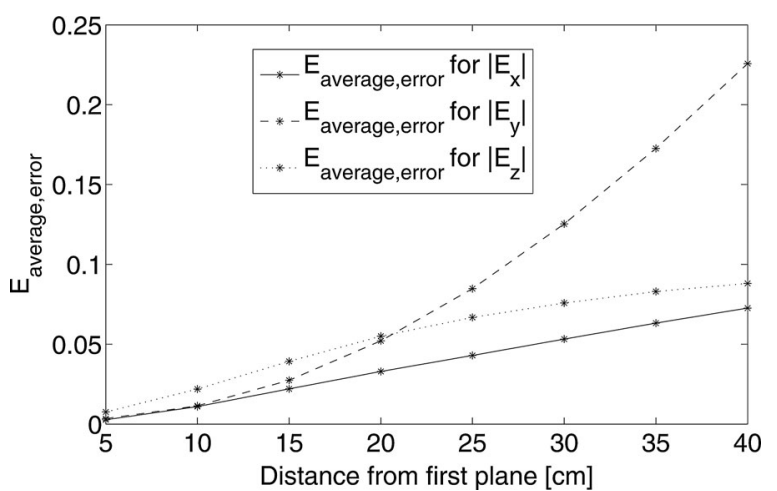

Fig. 6. The average of the absolute value for the amplitude error on each of the planes divided by the largest amplitude on the plane $E_{\text {average,error }}$ as function of distance to plane 1 .

The error $E_{\text {average,error at different distances from plane } 1 \text { is }}$ shown in Fig. 6.

The size of plane 1 for the here considered test case is $50 \mathrm{~cm} \times$ $50 \mathrm{~cm}$. We therefore conclude that it should be possible to obtain accurate field prediction for numerical dosimetry applications, with reasonable size for the measurement planes.

One way to avoid to use only the amplitudes and retrieved phase on one finite plane in the calculation of the field in the surrounding of the source, is to do measurements and phase retrieval on different sets of planes on different sides of the source. Combining the measurements on various planes, amplitude and phase could in principle be obtained on a closed surface around 

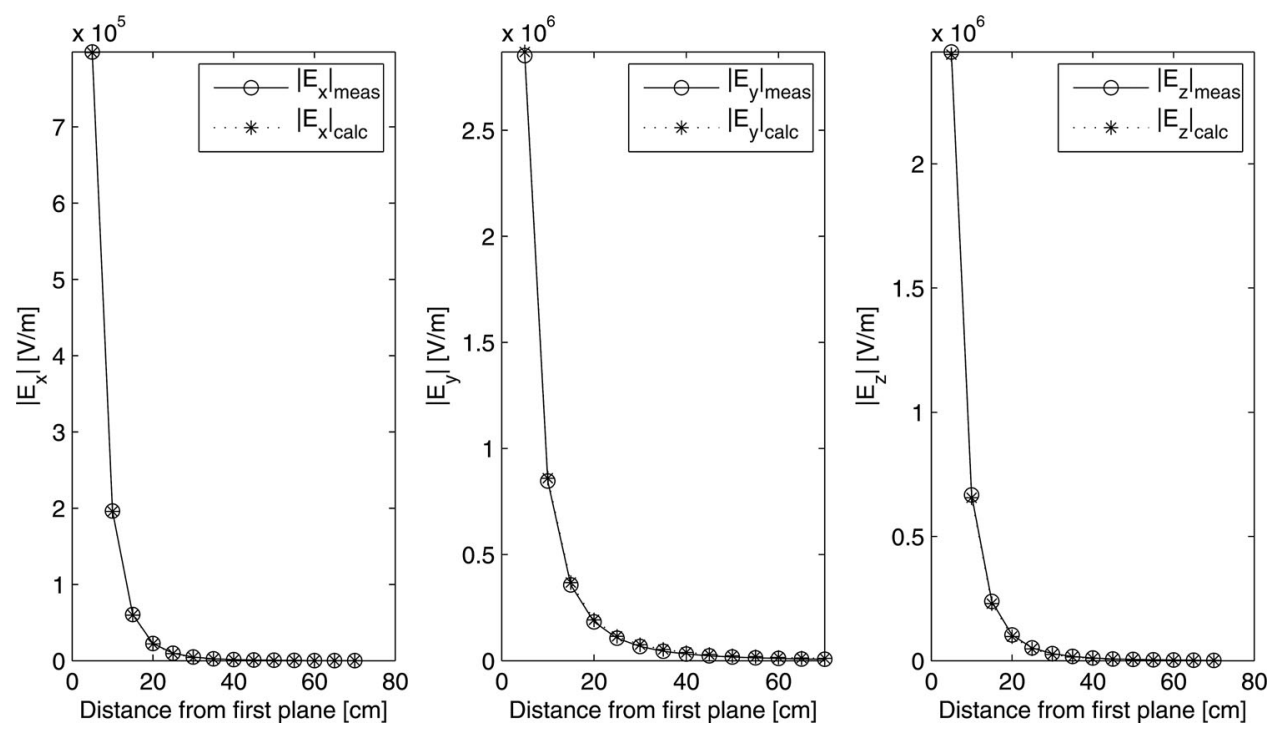

Fig. 7. Field amplitudes for the three Cartesian components as functions of distance to plane 1 . Both amplitude values calculated analytically $\left|E_{x}\right|$ m eas,$\left|E_{y}\right|$ m eas $\left|E_{z}\right|_{\text {meas }}$ and amplitudes calculated with retrieved phase $\left|E_{x}\right|_{\text {calc }},\left|E_{y}\right|_{\text {calc }},\left|E_{z}\right|_{\text {calc }}$ are shown. $\left|E_{x}\right|$ is shown for measurement points on the different planes with number 19 in $x$-direction and number 19 in $y$-direction. $\left|E_{y}\right|$ is shown for measurement points on the different planes with number 30 in $x$-direction and number 30 in $y$-direction. $\left|E_{z}\right|$ is shown for measurement points on the different planes with number 30 in $x$-direction and number 20 in $y$-direction. For the field components, measurement points with the $x$ - and $y$-coordinates where the field amplitude had its largest value on plane 2 were chosen

the source. Consequently, problems with the calculation of the field far away from the source or in certain directions could be avoided. In practice, however, it may in many situations be hard to do field measurements on all sides of the source. Depending on the situation it may be better to use one set of measurement planes or a few sets of planes pointing in different directions.

Next, the variation of the field amplitudes is illustrated in Fig. 7. The amplitudes for the three Cartesian field components are shown as functions of distance to plane 1. Both amplitude values calculated analytically $\left|E_{x}\right|_{\text {meas }},\left|E_{y}\right|_{\text {meas }},\left|E_{z}\right|_{\text {meas }}$ and amplitudes calculated with retrieved phase $\left|E_{x}\right|_{\text {calc }},\left|E_{y}\right|_{\text {calc }}$, $\left|E_{z}\right|_{\text {calc }}$ are shown. It can be seen that the calculated amplitudes the retrieved phase gave agree very well with the correct field. For each field component a measurement point where the value of the amplitude on plane 2 had its largest analytically calculated value was chosen. The amplitude value in that point, i.e., $\left(x^{\prime}, y^{\prime}, z_{1}\right)$, and the amplitudes in the points with the same $x$ and $y$-coordinates on the planes further away from the source $\left(x^{\prime}, y^{\prime}, z=z_{2}, z_{3} \ldots z_{14}\right)$ are shown in Fig. 7 .

\section{REsults for MEAsured Field VAlues}

The PAGM was also tested with measured field values. Measurements of the $50-\mathrm{Hz}$ field component of the magnetic flux density $\bar{B}$ in front of a transformer was performed. The amplitudes for all three Cartesian components of the field were measured. The measurements were performed with the measurement instrument BMM-3, which has a measurement probe with three perpendicular coils, one for each field component. The diameters of the coils are approximately $0.1 \mathrm{~m}$. The measurement inaccuracy for the instrument is according to manufacturer data within $\pm 5 \%$. A robot, ABB IRB1400, was used to

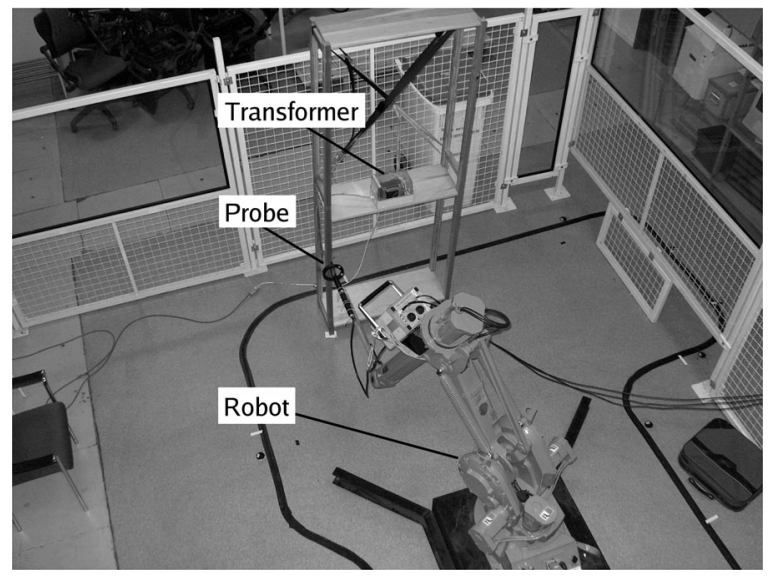

Fig. 8. Robot with measurement probe in front of a transformer.

move the measurement probe between the measurement points in front of the transformer (see Fig. 8).

\section{A. Phase Retrieval Based on Measured Magnetic Flux Density}

Measurement values from three vertical parallel planes (see Fig. 1), were used to calculate the phase angles. On plane 1, the number of measurement points was $161 \times 90$ and on the other two planes $151 \times 80$. Plane 1 was chosen larger than the other planes, to increase the accuracy in the calculations of the field on the other planes. The distances from plane 1 to plane 2 and from plane 2 to plane 3 were $5 \mathrm{~cm}$ and $2.5 \mathrm{~cm}$, respectively. The distance between the points on each of the three planes in horizontal as well as in vertical direction was $1 \mathrm{~cm}$. The obtained phase angles on plane 2 for $B_{x}$ and $B_{y}$, the field components tangential to the planes, are shown in Fig. 9. 


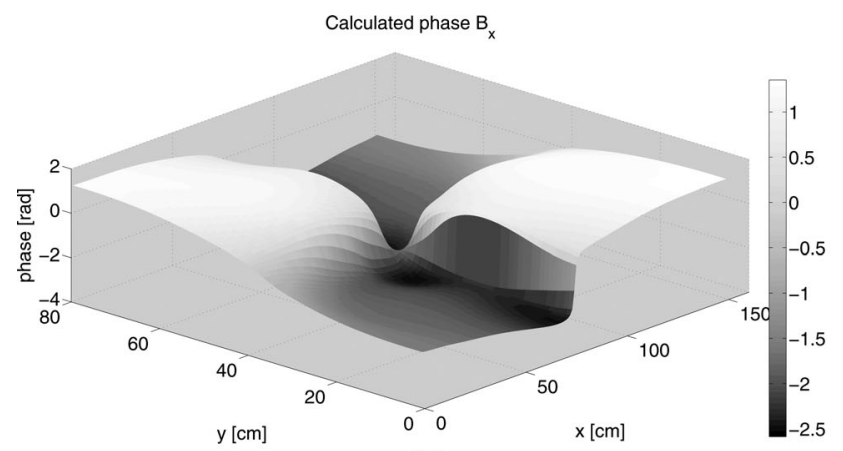

(a)

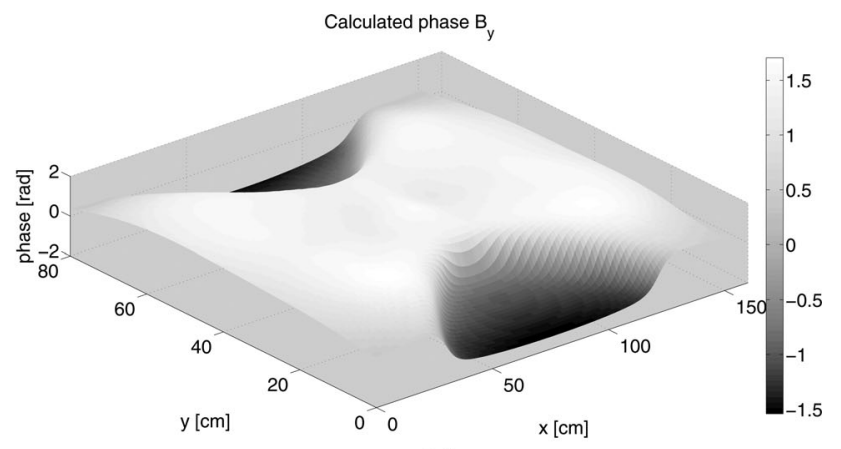

(b)

Fig. 9. Calculated phase angles using PAGM. (a) Phase angles $B_{x}$. (b) Phase angles $B_{y}$.

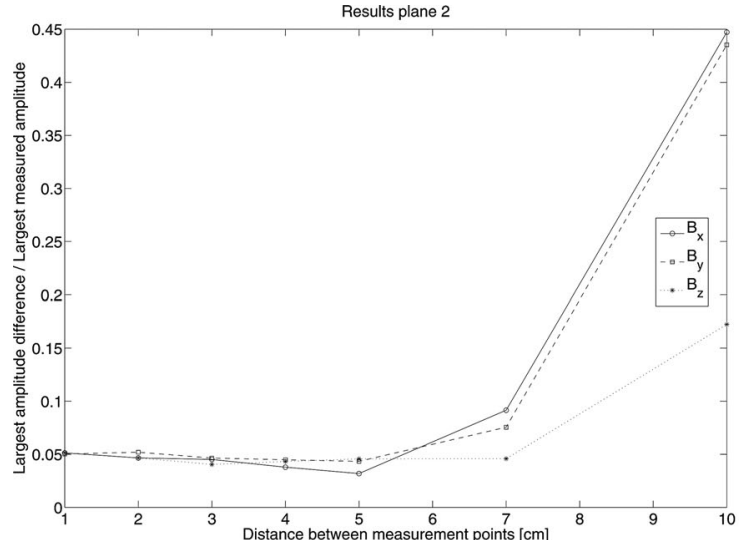

(a)

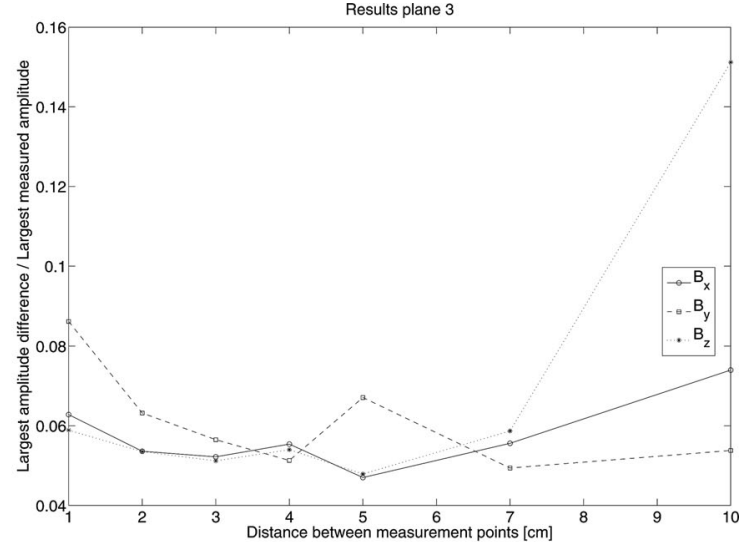

(b)

Fig. 10. Ratios between the largest amplitude difference and the largest measured amplitude for $B_{x}, B_{y}$ and $B_{z}$, as a function of distance between used measurement points. (a) For plane 2. (b) For plane 3.

In this situation, we do not have the correct phase from any analytical formula to compare with and the BMM-3 instrument, that was used for the measurements, does not provide information about the phase difference between the different measurement points. If the phase angles, given by the method, are similar to the correct ones, they should however give calculated field amplitudes that are similar to the measured amplitudes. Therefore, the phase angles that were obtained on plane 1, were used to calculate the amplitudes of $\bar{B}$ on the other planes. To test the number of points needed to give a good result, the method was tested for some different distances between the used measurement points on the planes. By omitting measurement points, the distance between the used points was varied.

The ratio between the largest amplitude difference and the largest measured amplitude $A_{\text {error }}$ for a field component on a plane can be defined as

$$
A_{\text {error }}=\frac{\left|a_{\text {calc }}-a_{\text {meas }}\right| \max }{a_{\text {meas }, \text { max }}}
$$

where $a_{\text {calc }}$ is calculated amplitude, $a_{\text {meas }}$ is measured amplitude and $a_{\text {meas,max }}$ is the largest measured amplitude on the plane. The value of $A_{\text {error }}$ on plane 2 are shown in Fig. 10(a) for $B_{x}, B_{y}$ and $B_{z}$, as a function of distance between used measurement points. The corresponding ratios for plane 3 can be found in Fig. 10(b). For the distances up to $7 \mathrm{~cm}$ stable results with small errors are obtained, but the distance $10 \mathrm{~cm}$ result in larger errors. In other sampling situations it can be so that the maximum sampling distance depend on how large the wavelength is. As mentioned before, however, the exponential function in (5) can be approximated with 1, for situations where the wavelength is much larger than the relevant distances. Thus, the maximum sampling distance in this situation should not depend much on the wavelength. It depends instead on how large the spatial variation for the field is, but it is hard to formulate it in any simple general sampling criteria.

\section{B. Spatial Variation of the Magnetic Flux Density}

For the distance $2 \mathrm{~cm}$ between used measurement points, field amplitudes on another plane, plane $4,2.5 \mathrm{~cm}$ further away from the source than plane 3 were also calculated. The measured amplitudes on this fourth plane were not used in the calculation of the phase angles. Fig. 11 shows measured $\left|B_{x}\right|$ on planes 3 and 4 . It can be seen that although the two planes are not so far from each other there is a clear difference (in terms of amplitude) between the measured field on planes 3 and 4. 


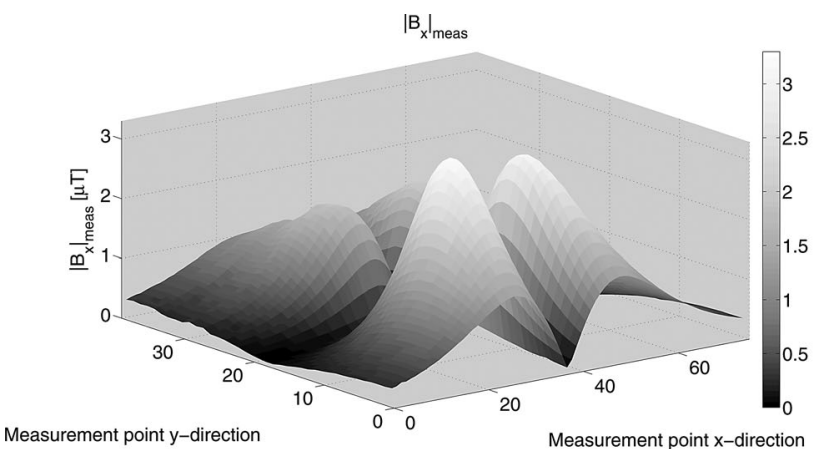

(a)

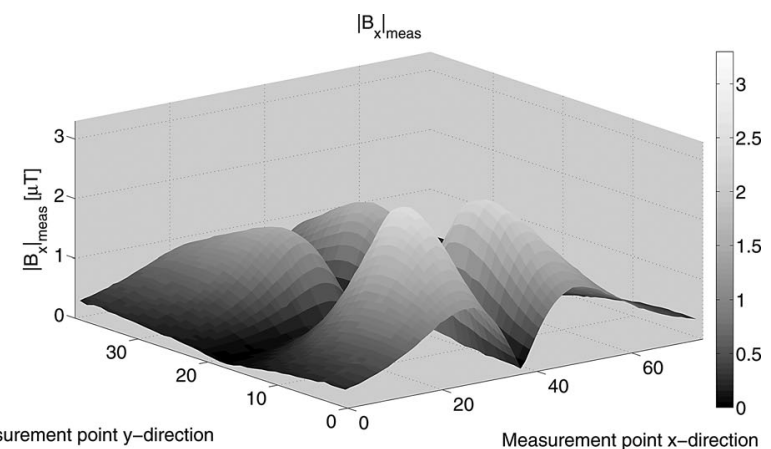

(b)

Fig. 11. Comparison between the field on plane 3 and plane 4. (a) Measured $\left|B_{x}\right|$ on plane 3. (b) Measured $\left|B_{x}\right|$ on plane 4.
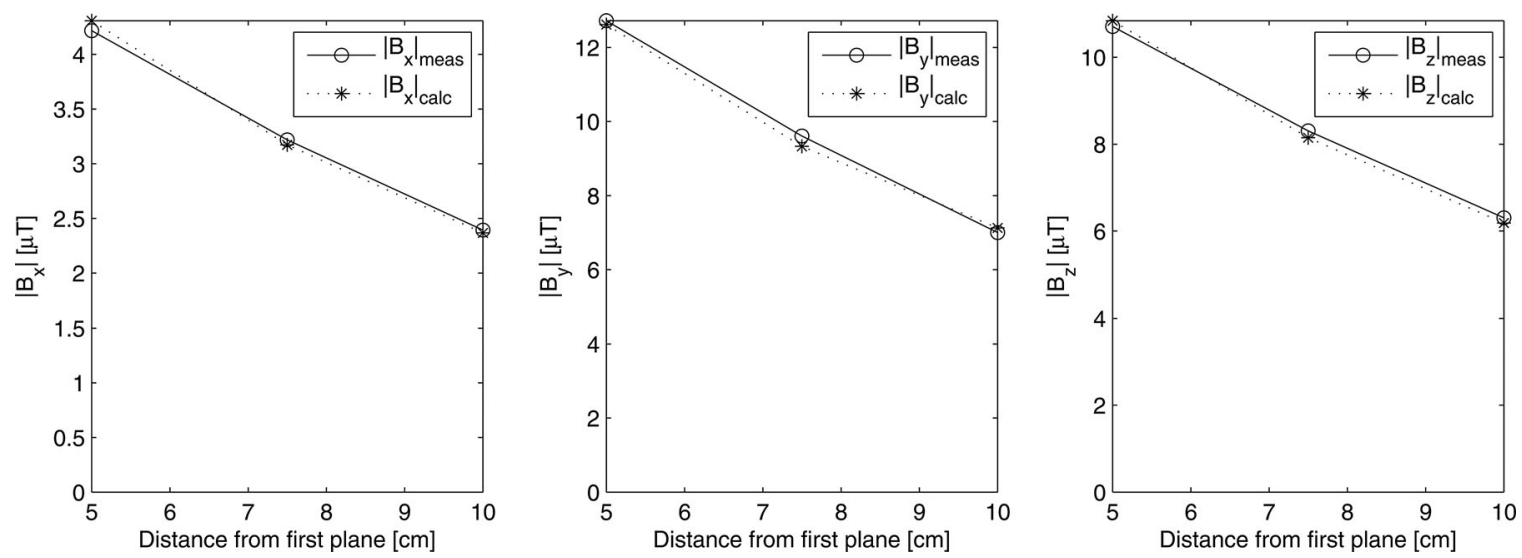

Fig. 12. Field amplitudes for the three Cartesian components as functions of distance to plane 1. Both measured amplitude values $\left|B_{x}\right|$ meas,$\left|B_{y}\right|$ meas, $\left|B_{z}\right|$ meas and amplitudes calculated with retrieved phase $\left|B_{x}\right|_{\text {calc }},\left|B_{y}\right|_{\text {calc }},\left|B_{z}\right|_{\text {calc }}$ are shown. $\left|B_{x}\right|$ is shown for measurement points on the different planes with number 31 in $x$-direction and number 13 in $y$-direction. $\left|B_{y}\right|$ is shown for measurement points on the different planes with number 38 in $x$-direction and number 19 in $y$-direction. $\left|B_{z}\right|$ is shown for measurement points on the different planes with number 38 in $x$-direction and number 13 in $y$-direction. For the field components, measurement points with the $x$ and $y$-coordinates where the measured field amplitude had its largest value on plane 2 were chosen.

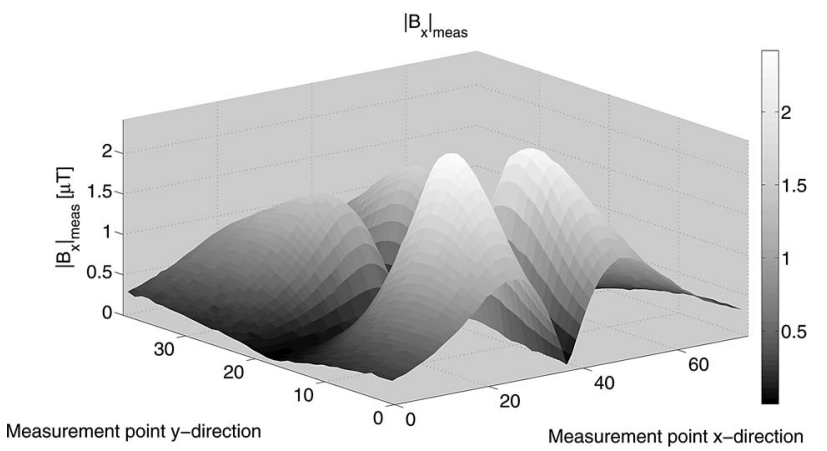

(a)

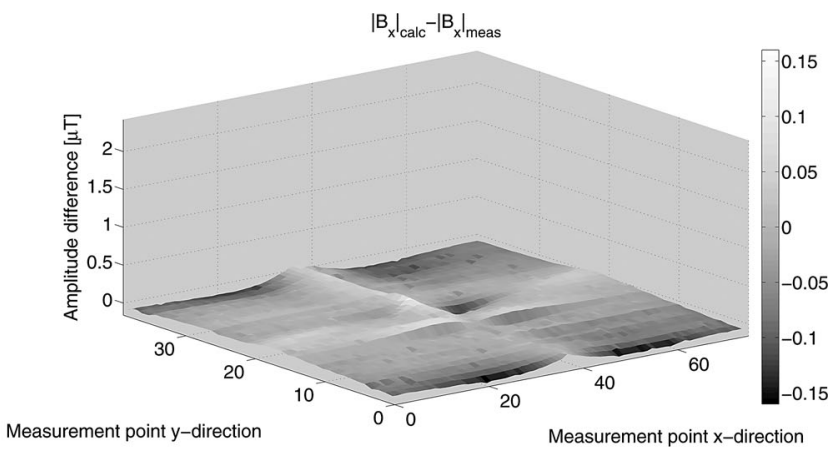

(b)

Fig. 13. Measured field amplitudes and differences between calculated and measured amplitudes on plane 4 .

The variation of the field distribution with distance is also illustrated in Fig. 12. The amplitudes for the three Cartesian field components are shown as functions of distance to plane 1. Both measured amplitude values $\left|B_{x}\right|_{\text {meas }},\left|B_{y}\right|_{\text {meas }},\left|B_{z}\right|_{\text {meas }}$ and amplitudes calculated with retrieved phase $\left|B_{x}\right|_{\text {calc }},\left|B_{y}\right|_{\text {calc }}$,
$\left|B_{z}\right|_{\text {calc }}$ are shown. It can be seen that the calculated amplitudes, the retrieved phase gave, agreed very well with the measured field. For each field component a measurement point where the value of the amplitude on plane 2 had its largest measured value was chosen. The amplitude value in that point and the values 


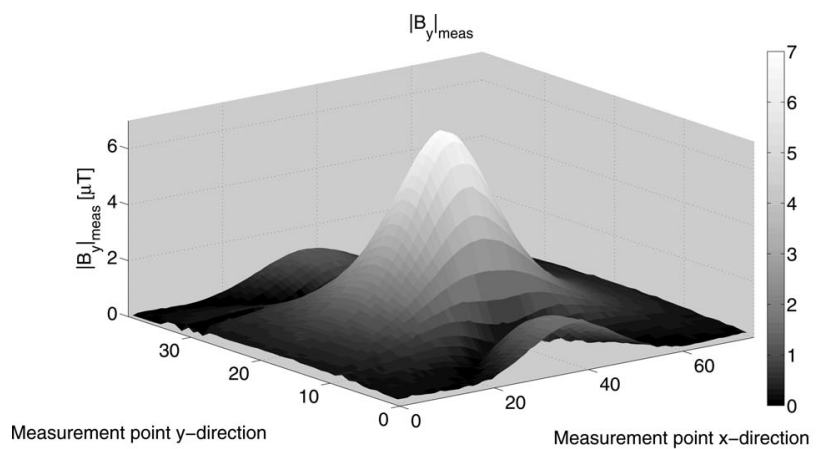

(a)

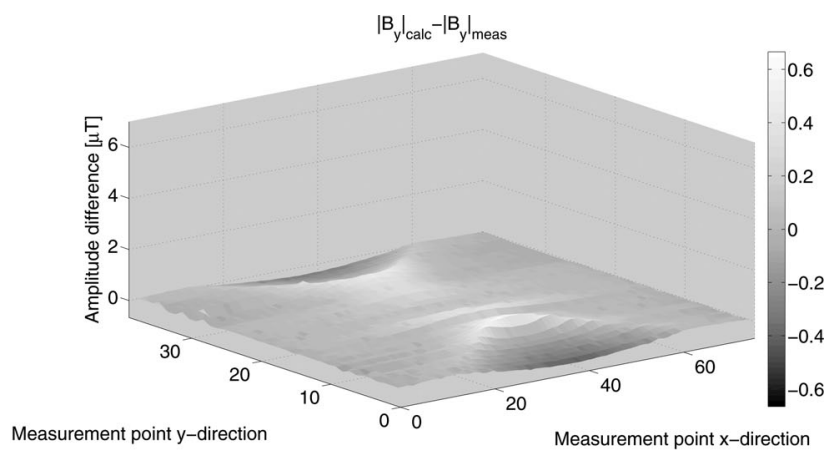

(b)

Fig. 14. Measured field amplitudes and differences between calculated and measured amplitudes on plane 4 .

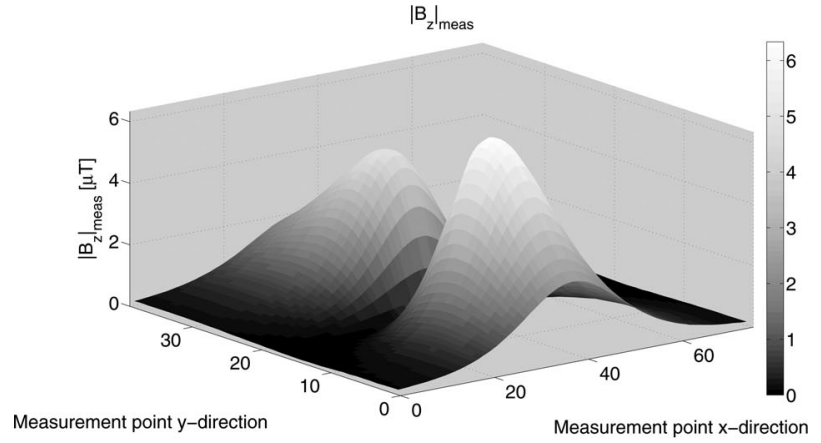

(a)

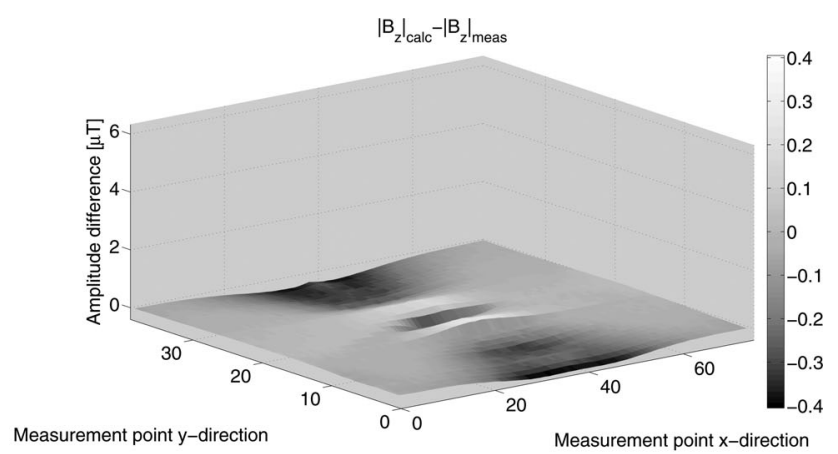

(b)

Fig. 15. Measured field amplitudes and differences between calculated and measured amplitudes on plane 4 .

in the points with the same $x$ - and $y$-coordinates on the planes further away from the source are the field amplitude values that are shown in Fig. 12.

In the Figs. 13-15, measured field amplitudes and the differences between calculated and measured amplitudes are shown for the different field components on plane 4. It can be seen that the differences between calculated and measured amplitudes are small. The ratios between the largest amplitude difference and the largest measured amplitude, as defined in (11), for $B_{x}$, $B_{y}$, and $B_{z}$ for this fourth plane are $6.62 \%, 9.51 \%$, and $6.40 \%$, respectively.

\section{CONCLUSION}

The results presented here together with previous ones in [14], [22], [23] show that the PAGM is very versatile, since it can be used for both high and low frequencies. The method gives excellent results for the numerical test cases and the transformer test case.

The numerical test cases showed that the method is very robust and that it works well even for test cases with plane sizes and separations between the planes that are extremely small in terms of wavelengths. It is quite insensitive to the initial guess for the phase distribution and poorly dependent on the location of the object under test with respect to the measurement planes. Such robustness is far from being guaranteed for other phase-retrieval techniques based either on functional minimization or iterative reconstruction. As compared to the method in [19], where the equivalent sources are found through successive comparison between the model predictions and the measurements, the PAGM is more automated and the equivalent sources do not need to be searched manually. Systematic trials have allowed determining experimental parameters of practical relevance such as the sampling space, the plane to plane distances, the testing zone as well as the reconstruction volume required to obtain a good accuracy.

These results have been validated by measurements conducted with the transformer test case at $50 \mathrm{~Hz}$. The retrieved phase angles on the measurement plane closest to the transformer gave calculated field amplitudes on other planes that agree well with the measured amplitudes. The ratios between the largest amplitude difference and the largest measured amplitude, as defined in (11), for $B_{x}, B_{y}$, and $B_{z}$ for the fourth plane are for example $6.62 \%, 9.51 \%$, and $6.40 \%$, respectively.

The PAGM is very useful for source modeling in situations where an electromagnetic source is complex or time consuming to make an accurate model of. It has the important advantage that it can be used for many different sources without a priori knowledge of the details. Since it can be used for distances that are small compared to the wavelength, the method is 
useful for both low-frequency EMC investigations and numerical dosimetry.

\section{ACKNOWLEDGMENT}

The authors humbly acknowledge that computations were performed on C3SE computing resources.

\section{REFERENCES}

[1] T. Isernia, G. Leone, and R. Pierri, "Radiation pattern evaluation from near-field intensities on planes," IEEE Trans. Antennas Propag., vol. 44, no. 5, pp. 701-710, May. 1996.

[2] F. Las-Heras and T. K. Sarkar, "A direct optimization approach for source reconstruction and NF-FF transformation using amplitude-only data," IEEE Trans. Antennas Propag., vol. 50, no. 4, pp. 500-510, Apr. 2002

[3] T. Isernia, G. Leone, and R. Pierri, "Results for a truncated phaseless near field technique," Electron. Lett., vol. 29, no. 5, pp. 505-506, Mar. 1993.

[4] T. K. Sarkar and F. Las-Heras, "A direct optimization approach for source reconstruction NF-FF using amplitude only data," in Proc. IEEE Int. Symp. Electromagn. Compat., 2001, vol. 1, pp. 399-402.

[5] F. Soldovieri, A. Liseno, G. D'Elia, and R. Pierri, "Global convergence of phase retrieval by quadratic approach," IEEE Trans. Antennas Propag., vol. 53, no. 10, pp. 3135-3141, Oct. 2005.

[6] R. W. Gerchberg and W. O. Saxton, "A practical algorithm for the determination of phase from image and diffraction plane pictures," Optik, vol. 35 , no. 2, pp. 237-246, 1972.

[7] A. Tennant, G. Junkin, and A. P. Anderson, "Antenna far-field predictions from two phaseless cylindrical near-field measurements," Electron. Lett., vol. 28, no. 23, pp. 2120-2122, Nov. 1992.

[8] R. G. Yaccarino and Y. Rahmat-Samii, "Phaseless bi-polar planar nearfield measurements and diagnostics of array antennas," IEEE Trans. Antennas Propag., vol. 47, no. 3, pp. 574-583, 1999.

[9] J. Fridén, M. Siegbahn, B. Thors, and L. Hamberg, "Quick SAR assessment using dual-plane amplitude-only measurement," presented at the EuCAP 2006, Nice, France.

[10] S. Farhad Razavi and Y. Rahmat-Samii, "A new look at phaseless planar near-field measurements: Limitations, simulations, measurements, and a hybrid solution," IEEE Antennas Propag. Mag., vol. 49, no. 2, pp. 170178, Apr. 2007.

[11] J. Fridén and H. Isaksson, "Robust phase-retrieval for quick SAR assessment using dual plane amplitude-only data," presented at the EMB 2007 Comput. Electromagn. Methods Appl. Conf. Proc., Lund, Sweden.

[12] J. R. Fienup, "Phase retrieval algorithms: A comparison," Appl. Opt., vol. 21, no. 15, pp. 2758-2769, 1982.

[13] A. Roczniak, E. M. Petriu, and G. I. Costache, "3-D electromagnetic field modeling based on near field measurements," Quality Measurements: The Indispensable Bridge Between Theory and Reality (No Measurements? No Science!) Joint Conf.-1996: IEEE Instrum. Meas. Technol. Conf. IMEKO Tech. Committee 7. Conf. Proc. (Cat. No.96CH35936), vol. 2, pp. 11241127.

[14] M. Johansson, L. E. Nord, R. Kopecký, A. Fhager, and M. Persson, "Computational methods for modeling of complex sources," COMPEL: Int. J. Comput. Math. Electr. Electron. Eng., vol. 27, no. 1, pp. 133-143, 2008.

[15] C. A. Balanis, Antenna Theory Analysis and Design. New York: Wiley, 1997.

[16] M. Rodríguez, M. Hernando, Y. Álvarez, F. Las-Heras, L. F. Herrán, and J. López, "Evaluation of the sources reconstruction technique applied to magnetic field measurement in power electronic circuits," in Proc. IEEE Power Electron. Spec. Conf., 2008 (PESC), pp. 529-534.

[17] M. M. Hernando, A. Fernández, M. Arias, M. Rodríguez, Y. Álvarez, and F. Las-Heras, "EMI radiated noise measurement system using the source reconstruction technique," IEEE Trans. Ind. Electron., vol. 55, no. 9, pp. 3258-3265, Sep. 2008

[18] M. Rodríguez, M. M. Hernando, M. Arias, Y. Álvarez, and F. Las-Heras, "Application of source reconstruction techniques and NF-FF transformations to estimate the EMI regulation compliance of a power electronic circuit," in Proc. 23rd Annu. IEEE Appl. Power Electron. Conf. Expo., 2008, pp. 1741-1746.

[19] D. Baudry, C. Arcambal, A. Louis, B. Mazari, and P. Eudeline, "Applications of the near-field techniques in EMC investigations," IEEE Trans. Electromagn. Compat., vol. 49, no. 3, pp. 485-493, Aug. 2007.
[20] S. Baillet, J. C. Mosher, and R. M. Leahy, "Electromagnetic brain mapping," IEEE Signal Process. Mag., vol. 18, no. 6, pp. 14-30, Nov. 2001.

[21] O. Chadebec, J.-L. Coulomb, G. Cauffet, J.-P. Bongiraud, and S. Guerin "Magnetization identification problem Illustration of an effective approach," COMPEL: Int. J. Comput. Math. Electr. Electron. Eng., vol. 23 , no. 2, pp. 518-530, 2004.

[22] M. Johansson, H. S. Lui, and M. Persson, "Performance evaluation of phase retrieval method based on amplitude-only near-field data," in Proc. Asia Pacific Microw. Conf. 2009, Singapore, Dec. 7-10,, pp. 425-428.

[23] M. Johansson, H.-S. Lui, and M. Persson, "Performance evaluation of phase-angle gradient method for phase retrieval based on low-frequency amplitude-only near-field data," Progr. Electromagn. Res. B, vol. 25, pp. 113-130, 2010.

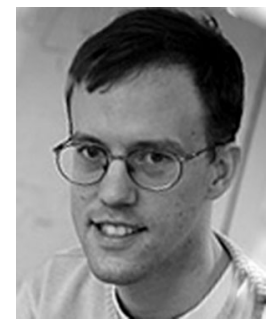

Markus Johansson received the M.Sc. degree in electrical engineering from The Royal Institute of Technology, Stockholm, Sweden, in 2003, and the Ph.D. degree from Chalmers University of Technology in Gothenburg, Sweden, in 2011.

His research interests include computational electromagnetics and dosimetry.

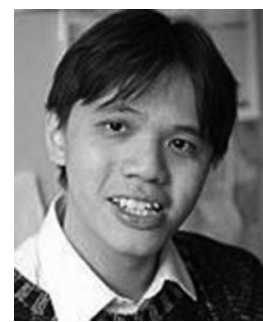

Hoi-Shun Lui (S'06-M'09) received the Bachelor of Engineering (first class Hons.) and Ph.D. degrees in electrical engineering from the University of Queensland, Brisbane, Australia, in 2003 and 2008, respectively.

While he was a Ph.D. candidate, he carried out consultancy work with Filtronics Australia Ltd. In 2008, he was a Research Fellow with the Microwave and RF Group at the National University of Singapore. He is now an Assistant Professor with the Department of Signals and Systems at Chalmers University of Technology, Gothenburg, Sweden. He also serves as a Project Leader in the VINN Excellence centre CHASE that is financed by the Swedish Governmental Agency for Innovation Systems (VINNOVA), industry and Chalmers. His research interest includes resonance-based radar target recognition, transient electromagnetic scattering, mutual coupling of antenna arrays and direction-ofarrival estimation.

Dr. Lui is an active reviewer in various international refereed journals such as IEEE TRANSACTIONS ON ANTENNAS AND PROPAGATIONS and IEEE ANTENNAS AND WIRELESS PROPAGATION LETTERS. 


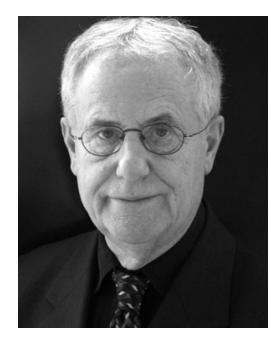

Jean-Charles Bolomey (M'99-SM'05-F'08) graduated from the Ecole Supérieure d'Electricité (Supelec), France, in 1963, and received the Ph.D. degree on an hybrid numerical-experimental approach to scattering in resonance domain from Paris-Sud University, France, in 1971.

$\mathrm{He}$ is currently an Emeritus Professor at ParisSud University. He became a Professor at Paris-Sud University, in 1976. He received the Grade Emeritus of SEE, in 1995. His research has been conducted in the Electromagnetic Research Department of the Laboratoire des Signaux et Systèmes, a joint unit of Supelec and the National Center for Scientific Research (CNRS). Since 1981, his research contributions have been devoted to Near-Field techniques in a broad sense, including antenna measurement, EMC testing, as well as Industrial-Scientific-Medical (ISM) applications. These contributions have largely concerned measurement techniques and have been deliberately oriented toward innovative technology transfer and valorization. He has more particularly promoted the modulated probe array technology, demonstrating its unrivaled potential for rapid Near-Field scanning. He has coauthored with Prof. F. Gardiol a reference book on principles and applications of the Modulated Scattering Technique (MST). He is holder of numerous patents covering various MST-based probe array arrangements for microwave sensing and imaging systems. In 1986, under the impulse of the National Agency for Valorization (ANVAR) and of the CNRS, he founded the company SATIMO, which is now considered as a leading company in the field of antenna measurement. He has been also involved in industrial applications of microwave heating as a Chairman of the Microwave Group of Electricité de France (EDF) and was appointed as a Consultant by the Délégation Générale de l'Armement (DGA) in the field of High Power Microwave (HPM) metrology. He has also actively contributed to several cooperative European programs ranging from medical hyperthermia to industrial process tomography and has contributed to various prototype transfer and evaluation procedures in these areas. Recently, his research was related to RF dosimetry and rapid SAR measurements for wireless communication devices. He is now continuing his research on load-modulated scattering antennas, and, more particularly, novel sensing applications of RFID technology. He is also contributing as a member of several Scientific Advisory Boards of European Institutions (Chalmers University, Queen Mary London University) and startup companies. He has been appointed as Distinguished Lecturer of the IEEE Antennas and Propagation Society (2011-2013).

Prof. Bolomey has received several awards, including the Blondel Medal of the Société des Electriciens et des Electroniciens (SEE), in 1976, the Général Ferrié Award of the French Academy of Sciences, in 1984, and the Best Paper Award of the European Microwave Conference (EuMC), in 1983. In 1994, he was granted the Schlumberger Stitching Fund Award for his contribution to inverse scattering techniques in microwave imagery. In 2001, he received the Distinguished Achievement Award of the Antenna Measurement Technique Association (AMTA) for his pioneering activity in the field of modulated probe arrays, and in 2007, elected as Edmond S. Gillespie Fellow for AMTA. He received the 2004 Medal of the French URSI Chapter. He has obtained the 2006 H.A. Wheeler Best Application Prize Paper Award of the IEEE AP-Society for his coauthored paper on "Spherical Near-Field Facility for Characterizing Random Emissions."

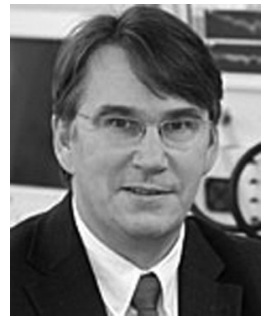

Mikael Persson (M'10) received the M.Sc. and Ph.D. degrees from Chalmers University of Technology, Göteborg, Sweden, in 1982 and 1987, respectively.

$\mathrm{He}$ is a Professor of biomedical electromagnetics at Chalmers University of Technology, Göteborg, Sweden. He is the author or coauthor of more than 100 journal and conference papers in biomedical electromagnetics and fusion modeling.

Prof. Persson is the present member of the Swedish Committee (2001-2002)
URSI and has served on the European Fusion Physics 